\title{
On comparing estimates of low-I solar p-mode frequencies from Sun-as-a-star and resolved observations
}

\author{
W. J. Chaplin ${ }^{1}$, T. Appourchaux ${ }^{2, \star}$, Y. Elsworth ${ }^{1}$, G. R. Isaak ${ }^{1}$, B. A. Miller ${ }^{1}$, and R. New ${ }^{3}$ \\ 1 School of Physics and Astronomy, University of Birmingham, Edgbaston, Birmingham B15 2TT, UK \\ e-mail: wjc@bison.ph.bham.ac.uk \\ 2 ESA Research and Science Support Department, ESTEC, 2200 AG Noordwijk, The Netherlands \\ 3 School of Science \& Mathematics, Sheffield Hallam University, Sheffield S1 1WB, UK
}

Received 23 March 2004 / Accepted 5 July 2004

\begin{abstract}
Low-angular-degree (low- $l$ ) solar p modes provide a sensitive probe of the radiative interior and core of the Sun. Estimates of their centroid frequencies can be used to constrain the spherically symmetric structure of these deep-lying layers. The required data can be extracted from two types of observation: one where the modes are detected in integrated sunlight, i.e., a Sun-as-a-star view; and a second where the visible disc is imaged onto many pixels, and the collected images then decomposed into their constituent spherical harmonics. While the imaging strategy provides access to all of the individual components of a multiplet, the Sun-as-a-star technique is sensitive to only about two thirds of these (average over $l=0$ to 3 ) with those modes that are detected having different levels of visibility. Because the various components can have contrasting spatial structure over the solar surface, they can respond very differently to changes in activity along the solar cycle. Since the Sun-as-a-star and resolved analyses take as input a different "subset" of modes, the extracted frequency estimates are expected to differ depending upon the phase of the cycle. Differences also arise from the types of models used to fit the modes. Here, we present expressions that allow the sizes of these differences to be predicted.
\end{abstract}

Key words. methods: data analysis - Sun: helioseismology

\section{Introduction}

The structure of the deep radiative interior of the Sun can be inferred from observational estimates of the frequencies of core-penetrating, low-angular-degree (low- $l$ ) solar $\mathrm{p}$ modes. This is accomplished by inversion of the frequencies (Christensen-Dalsgaard 2002). Estimates of the low-degree parameters come from two "types" of observation. In the first, the Doppler velocity or intensity perturbations associated with the modes are averaged over the visible disc by observations that detect them in integrated sunlight, i.e., a full-disc [FD] or "Sun-as-a-star" view. Examples of this type of observation include the ground-based Birmingham SolarOscillations Network (BiSON; Aindow et al. 1988; Chaplin et al. 1996) and more recently the GOLF (Gabriel et al. 1995) and VIRGO/SPM (Fröhlich et al. 1997) instruments on board the ESA/NASA SOHO satellite. In the second, the solar disc is imaged over many pixels. The collected images are then

^ Now at: Institut d'Astrophysique Spatiale, Bâtiment 121, 91045, Orsay Cedex, Paris, France. decomposed into their constituent spherical harmonics. For each overtone, $n$, and degree $l$, this gives $2 l+1$ spectra (where $m$ is the azimuthal order, and $-l \leq m \leq l$ ). Resolved [RES] observations are currently being made by, amongst others, the ground-based Global Oscillations Network Group (GONG; Harvey et al. 1996) the two-site LOWL/ECHO (Tomczyk et al. 1995), and the MDI (Scherrer et al. 1995) and VIRGO/LOI (Appourchaux et al. 1995) instruments on board SOHO.

Inversions and comparisons are often made using combinations of Sun-as-a-star and resolved low-l frequencies (e.g., Basu et al. 1997, 2000; Couvidat et al. 2003). However, even when contemporaneous data are used, the frequencies represent estimates of different underlying quantities, i.e., there is an inherent bias between the two sets. This reflects two things: first, the different way in which the two types of estimates are affected by changes in activity over the solar cycle; and to a lesser extent, the manner in which the data are analyzed to extract estimates of the frequencies.

The activity-dependent differences can be a cause for concern. Linear inversion formalism expresses the small 
differences between observed and modelled frequencies as arising from a linear combination of differences in the interior sound speed and density, with an additional "surface" term that allows for inadequacies in modelling the near-surface layers (e.g., departures from adiabaticity; see Christensen-Dalsgaard et al. 2000 for an in-depth discussion). This term is usually regarded as being spherically symmetric. As such, any $l$ dependence comes in only through the variation of mode inertia with angular degree. However, the differences we discuss here arise from non-spherically-symmetric effects which are sensitive to the azimuthal projection of the mode, $m$. Our aim in this contribution is to formalize the differences in terms of quantities that are measurable over the activity cycle and to provide a useful reference for those less familiar with procedures used to extract mode frequencies.

\section{What is a "resolved" or "Sun-as-a-star/Full Disc" mode frequency?}

\subsection{Resolved frequency estimates}

The full set of $m$ frequencies extracted from resolved-Sun observations are usually described using a polynomial expansion of the form:

$v_{n, l, m}=v_{n, l}^{\mathrm{RES}}+\sum_{j=1}^{j_{\max }} a_{j}(n, l) l \mathcal{P}_{l}^{j}(m)$,

where $j_{\max } \leq 2 l$, and the basis functions are polynomials related to Clebsch-Gordan coefficients (Ritzwoller \& Lavely 1991). The first six of these are given in Appendix A. In effect, each component has an equal weighting in determining the terms of the expansion.

The offset term in the expansion, $v_{n, l}^{\text {RES }}$, is the so-called central frequency of the multiplet (i.e., its centroid). Here, we tag it with "RES" to show it comes from the resolved-Sun data. It is these centroid frequencies that are used as input to inversions to find the spherically symmetric component of the internal structure. The odd $a_{j}$ coefficients describe spherically symmetric contributions to the splittings within a multiplet, e.g., from the internal rotation; while the even $a_{j}$ reflect perturbations that are non-spherically symmetric in nature, with the largest contribution coming from the influence of the spatially inhomogeneous magnetic activity over the solar surface. While temporal variations in the low-order odd $a_{j}$ remain undetected (i.e., $a_{1}, a_{3}$ and $a_{5}$ ), significant changes have been uncovered in both the $v_{n, l}^{\text {RES }}$ and even $a_{j}$ that are well-correlated with the solar activity cycle (e.g., Dziembowski et al. 2000; Antia et al. 2001; Howe et al. 2002; Appourchaux et al. 2002).

The upper panel of Fig. 1 shows an illustrative example of the distribution of components one might expect in the $l=2$, $n=21$ overtone at $\sim 3160 \mu \mathrm{Hz}$ under conditions of high surface activity. In order to construct the figure we used $a_{1}=0.4 \mu \mathrm{Hz}$, $a_{2}=0.1 \mu \mathrm{Hz}$, and $a_{3} \simeq a_{4} \simeq 0$. These correspond to typical, average $l=2$ values extracted from 1-yr GONG or LOI data for the 1-yr (high-activity) period beginning 2000 March 27. Each symbol corresponds to a different $m$ (as indicated on the ordinate), with the location of the datum on the abscissa corresponding to the frequency offset of the component (here, with
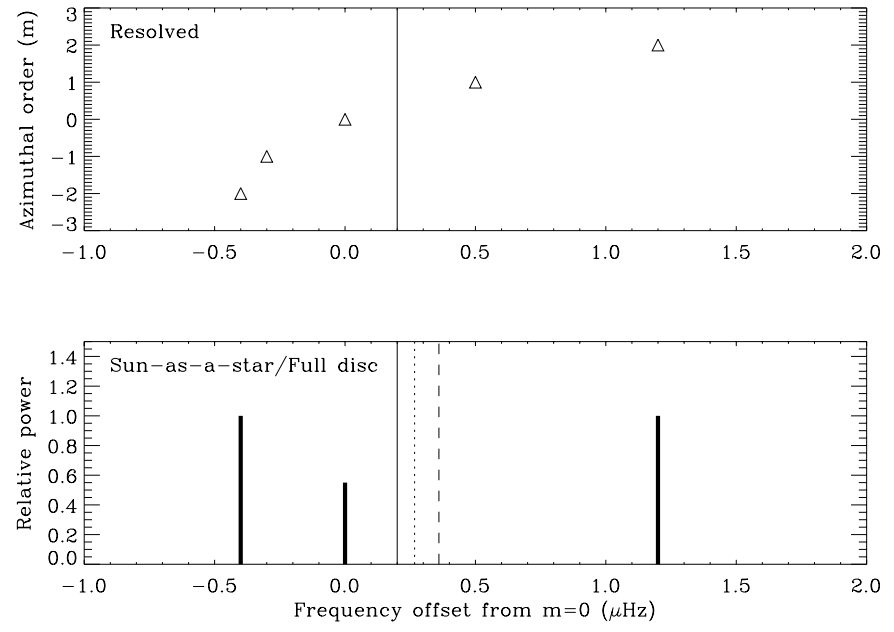

Fig. 1. Upper panel: distribution of components expected in resolved data - when all $m$ can be detected - for the $l=2, n=21(\sim 3160 \mu \mathrm{Hz})$ mode at levels of high activity. Each symbol corresponds to a given $m$ (as indicated on the ordinate), whose location on the abscissa corresponds to the frequency offset of the component (here, with respect to the location of the $m=0$ component). The solid vertical line shows the location of the centroid frequency $v_{n, l}^{\mathrm{RES}}$. Lower panel: frequency locations of the three $l=2$ components visible in Sun-as-a-star data (thick bars). The vertical dashed $\left(v_{n, l}^{\mathrm{FD} t r a d}\right)$ and dotted $\left(v_{n, l}^{\mathrm{FDcen}}\right)$ lines are the Sun-as-a-star frequencies extracted by fitting two types of model (see text for more details). The solid vertical line again shows the resolved-data centroid for comparison.

respect to the location of the $m=0$ ). The solid vertical line shows the location of the centroid frequency $v_{n, l}^{\text {RES }}$.

In summary, since every component can be observed the multiplet frequencies, $v_{n, l}^{\mathrm{RES}}$, reflect the contribution of all $2 l+1$ modes. As we shall see, this is not the case for Sunas-a-star observations.

\subsection{Sun-as-a-star frequency estimates}

The visibility of a mode observed in integrated sunlight depends upon the average of its displacement pattern over the disc, and the projection of this with respect to the preferred axis of the system, i.e., that of the solar rotation. When observations are made in or close to the ecliptic plane - meaning that the rotation axis is at near right-angles - only those components for which $l+m$ is even are clearly visible. Furthermore, the sensitivity to different azimuthal orders means that at $l \geq 1$ the outer components are observed to be the most prominent. This is the perspective offered from both ground-based and SOHO-borne instruments.

Since not all the constituent components are seen, and those that are have different visibilities, mode frequencies derived from such data are weighted strongly by the contribution from particular $|m|$. As noted above, it is those with $|m|=l$ that carry by far the largest weight. At $l=1$ it is only these components that are seen. In spectra made from BiSON data the $l=2$, $|m|=2$ peaks are a factor of 1.8 larger in power than their $m=0$ neighbours; while at $l=3$, the outer components are 
2.6-times stronger than the $|m|=1$ peaks. This is illustrated for an $l=2$ mode in the lower panel of Fig. 1. The three solid bars show the locations of the observed components (offset with respect to $m=0$ ), with the height on the ordinate a measure of the relative power seen in a BiSON frequency spectrum.

A set of Sun-as-a-star observations gives a single time series whose frequency spectrum will contain many closely spaced resonant peaks. This means that any fitting strategy must fit the components of a multiplet simultaneously. Furthermore, when $l \geq 2$ those peaks that are visible will be distributed unevenly in frequency at moderate to high levels of activity. Because of the close spacing, and the relative weakness of the inner components, it is very difficult to fix or determine this distribution reliably. This is why it has long been standard practice to fit a model that takes no account of the uneven spacing. As such, this "traditional" fitting method assumes the visible components are spaced symmetrically in frequency. We shall refer to estimates extracted in this manner as $v_{n, l}^{\text {FDtrad }}$. The vertical dashed line in the lower panel of the Fig. 1 shows the frequency that would be derived from fitting such a model to the $l=2$ mode as shown (see Sect. 3.2 for an explanation of the location). For comparison, the resolved-data centroid, $v_{n, l}^{\mathrm{RES}}$, is shown as the vertical unbroken line. The two clearly do not coincide.

To develop a more refined estimate for the full-disc frequency, Chaplin et al. (2003) have shown that one can attempt to take account of the uneven spacing provided the dataset is of sufficient length (thereby giving the required resolution). This in effect allows one to estimate the locations of each of the component frequencies. A value for the centroid (i.e., straight forward average) of the visible Sun-as-a-star/full-disc frequencies can then be derived. This we refer to as $v_{n, l}^{\text {FDcen }}$. This is rendered as a vertical dotted line for the example in Fig. 1; again, there is an offset with respect to the "resolved" $v_{n, l}^{\text {RES }}$ (see Sect. 3.2).

\section{Difference in frequency estimates}

The treatment that follows takes no account of potential bias introduced by temporal (from the observational window function) and spatial (from the resolved-data decomposition) leakage. Furthermore, we do not consider small differences in frequency that can arise from offsets in mode-peak asymmetry between datasets. Various studies have shown that this has a contribution that depends upon the nature of the observing technique (e.g., Duvall et al. 1993; Toutain et al. 1997, 1998; Chaplin \& Appourchaux 1999; Howe et al. 2003). On the assumption that allowance has been made for these and other potential sources of analysis bias, we would expect the Sunas-a-star and resolved estimates of the radial $(l=0)$ mode frequencies to be measures of the same underlying parameter (provided the data are contemporaneous). However, at $l \geq 1$ we must allow for the influence of the changing activity. In what follows we present expressions to relate the full-disc to the resolved-data centroid frequencies. We have checked these predictions using 1-yr spectra made from BiSON and LOI data, and found the extracted results to be consistent with the expressions at the level of precision of the observations.

\subsection{Dipole $(I=1)$ modes}

The frequencies of the dipole components visible in Sun-asa-star data can be written explicitly in terms of the resolveddata centroid frequency and the $a_{j}$ coefficients (see Eq. (1) and Appendix A), i.e.,

$v_{n, 1,1}=v_{n, 1}^{\mathrm{RES}}+a_{1}(n, 1)+a_{2}(n, 1)$,

and

$v_{n, 1,-1}=v_{n, 1}^{\mathrm{RES}}-a_{1}(n, 1)+a_{2}(n, 1)$.

Since only two components are seen in the full-disc data, the extracted frequency estimate will be the simple average of the corresponding frequencies, i.e.,

$v_{n, 1}^{\mathrm{FDtrad}}=v_{n, 1}^{\mathrm{RES}}+a_{2}(n, 1)$.

The "traditional" fitting method provides the necessary means of obtaining this. The difference between the full-disc and resolved frequencies is dependent upon the magnitude of the $a_{2}$ coefficient. For an $l=1$ mode near the centre of the p-mode spectrum this may be as large as $\approx 0.1 \mu \mathrm{Hz}$ at times of high activity.

\subsection{Quadrupole $(I=2)$ modes}

At $l=2$, the three components observed in Sun-as-a-star data are:

$v_{n, 2,2}=v_{n, 2}^{\mathrm{RES}}+2 a_{1}(n, 2)+2 a_{2}(n, 2)+2 a_{3}(n, 2)+2 a_{4}(n, 2),(5)$

$v_{n, 2,0}=v_{n, 2}^{\mathrm{RES}}-2 a_{2}(n, 2)+12 a_{4}(n, 2)$,

and

$v_{n, 2,-2}=v_{n, 2}^{\mathrm{RES}}-2 a_{1}(n, 2)+2 a_{2}(n, 2)-2 a_{3}(n, 2)+2 a_{4}(n, 2)$.

The presence of the extra central component - which may be distributed unevenly in frequency between the outer components - now complicates the fitting. As noted in Sect. 2.2, different frequency estimates can be extracted dependent upon the nature of the fitting model. In the "traditional" method, the model assumes the $m=0$ peak lies symmetrically between the outer peaks. Under circumstances where all even $a_{j}$ are zero, this provides an accurate description and the fitted frequency will then be the mean of the locations of all three visible components; more importantly for the practicalities of the fitting procedure, it will of course also be the mean of the two stronger $|m|=2$ components which dominate the observed profile.

The presence of active regions distributed in a nonspherically symmetric manner over the surface will give rise to non-zero $a_{j}$, so that the peaks are distributed unevenly in frequency. The symmetric model is then no longer an accurate representation of the underlying structure; if used, the fitted value it returns will be "pulled" by the inner, weaker component in the direction of the outer peak that lies closest to it. The extracted value is therefore no longer the mean of the outerpeak frequencies, but some linear combination of these and the $m=0$ frequency. 
If $\mathcal{S}_{n, l}$ is the fractional contribution of the $(2,0)$ component, the fitted $v_{n, 2}^{\mathrm{FDtrad}}$ can be represented by

$$
v_{n, 2}^{\mathrm{FD} \text { trad }}=\mathcal{S}_{n, 2} \cdot v_{n, 2,0}+\left(1-\mathcal{S}_{n, 2}\right) \cdot\left[\frac{v_{n, 2,2}+v_{n, 2,-2}}{2}\right]
$$

Chaplin et al. (2004) showed that the factor $\mathcal{S}_{n, 2}$ is fairly constant over the main part of the p-mode spectrum $(2000 \lesssim v \lesssim$ $3500 \mu \mathrm{Hz}$ ) where the modes are most prominent. They found that for BiSON-like data it takes the value $\mathcal{S}_{n, 2} \sim 0.1$; outside this region the factor decreases in size. So, even though a $(2,0)$ mode has a height in the power spectrum that is $\sim 55 \%$ of that of its $(2,2)$ neighbours, its influence on the fitted frequency is somewhat less than would be implied by a simple linear scaling with the visibility ratio.

Given Eqs. (5)-(7) - which describe the individual component frequencies - it then follows that the "traditional" frequency will be related to the resolved-data centroid by:

$$
v_{n, 2}^{\mathrm{FDtrad}}=v_{n, 2}^{\mathrm{RES}}+\left(2-4 \mathcal{S}_{n, 2}\right) \cdot a_{2}(n, 2)+\left(2+10 \mathcal{S}_{n, 2}\right) \cdot a_{4}(n, 2) .
$$

For a mode at the centre of the spectrum the difference between $v_{n, l}^{\text {RES }}$ and $v_{n, 2}^{\text {FDtrad }}$ may therefore be as large as $\approx 0.13 \mu \mathrm{Hz}$ at times of high activity (when for $l=2$ the $a_{2}$ is $\approx 0.08 \mu \mathrm{Hz}$ at $3000 \mu \mathrm{Hz}$ ).

Provided the dataset is of sufficient length an attempt can be made to fit for the locations of all three Sun-as-a-star components. If the frequency thereby extracted, $v_{n, 2}^{\text {FDcen }}$, corresponds to the mean (unweighted centroid) of the three visible modes we have:

$$
\begin{aligned}
v_{n, 2}^{\mathrm{FDcen}} & =\left[v_{n, 2,2}+v_{n, 2,0}+v_{n, 2,-2}\right] / 3 \\
& =v_{n, 2}^{\mathrm{RES}}+0.67 a_{2}(n, 2)+5.33 a_{4}(n, 2) .
\end{aligned}
$$

The difference between this and the resolved-Sun centroid can rise to $\approx 0.05 \mu \mathrm{Hz}$ (again for a mode near $\approx 3000 \mu \mathrm{Hz}$ ).

\subsection{Octupole $(I=3)$ modes}

At $l=3$, the four components seen in Sun-as-a-star data are:

$$
\begin{aligned}
v_{n, 3,3}= & v_{n, 3}^{\mathrm{RES}}+3 a_{1}(n, 3)+3 a_{2}(n, 3)+3 a_{3}(n, 3)+3 a_{4}(n, 3) \\
& +3 a_{5}(n, 3)+3 a_{6}(n, 3) \\
v_{n, 3,1}= & v_{n, 3}^{\mathrm{RES}}+a_{1}(n, 3)-1.8 a_{2}(n, 3)-3 a_{3}(n, 3) \\
& +a_{4}(n, 3)+15 a_{5}(n, 3)+45 a_{6}(n, 3) \\
v_{n, 3,-1}= & v_{n, 3}^{\mathrm{RES}}-a_{1}(n, 3)-1.8 a_{2}(n, 3)+3 a_{3}(n, 3) \\
& +a_{4}(n, 3)-15 a_{5}(n, 3)+45 a_{6}(n, 3)
\end{aligned}
$$

and

$$
\begin{aligned}
v_{n, 3,-3}= & v_{n, 3}^{\mathrm{RES}}-3 a_{1}(n, 3)+3 a_{2}(n, 3)-3 a_{3}(n, 3)+3 a_{4}(n, 3) \\
& -3 a_{5}(n, 3)+3 a_{6}(n, 3)
\end{aligned}
$$

Again, two fitting strategies can be adopted. For the traditional model $\mathcal{S}_{n, 3}$ is now the fractional contribution of the $(3,1)$ and $(3,-1)$ frequencies to the traditional-model frequency, i.e.,

$$
\begin{aligned}
v_{n, 3}^{\mathrm{FD} \text { trad }}= & \mathcal{S}_{n, 3} \cdot\left[\frac{v_{n, 3,1}+v_{n, 3,-1}}{2}\right] \\
& +\left(1-\mathcal{S}_{n, 3}\right) \cdot\left[\frac{v_{n, 3,3}+v_{n, 3,-3}}{2}\right] .
\end{aligned}
$$

The extracted frequency is then given by

$$
\begin{aligned}
v_{n, 3}^{\mathrm{FD} t r a d} & =v_{n, 3}^{\mathrm{RES}}+\left(3-4.8 \mathcal{S}_{n, 3}\right) \cdot a_{2}(n, 3)+\left(3-2 \mathcal{S}_{n, 3}\right) \cdot a_{4}(n, 3) \\
& +\left(3+42 \mathcal{S}_{n, 3}\right) \cdot a_{6}(n, 3) .
\end{aligned}
$$

The constant $\mathcal{S}_{n, 3} \sim 0.06$ for BiSON data. For a mode at the centre of the spectrum the difference between $v_{n, 3}^{\mathrm{RES}}$ and $v_{n, 2}^{\mathrm{FDtrad}}$ may therefore be as large as $\approx 0.14 \mu \mathrm{Hz}$ at times of high activity (when for $l=3$ the $a_{2} \approx 0.05 \mu \mathrm{Hz}$ ).

The second strategy, which attempts to fix the precise locations of all four peaks, is much more difficult to apply at $l=3$. Indeed, a robust determination of the underlying distribution of peaks has not yet been extracted from Sun-as-a-star data. This is because the strongest, $|m|=3$ peaks have heights that are only $\sim 36 \%$ of that of their $l=2,|m|=2$ counterparts (BiSON data). In addition, the height contrast between the inner and outer peaks is greater than for the quadrupole modes. Nevertheless, we include here an expression relating the unweighted mean of the four visible Sun-as-a-star components - an estimate that could be extracted from the successful application of an uneven-distribution fitting model - to the resolved-data centroid:

$$
\begin{aligned}
v_{n, 3}^{\mathrm{FDcen}} & =\left[v_{n, 3,3}+v_{n, 3,1}+v_{n, 3,-1}+v_{n, 3,-3}\right] / 4 \\
& =v_{n, 3}^{\mathrm{RES}}+0.6 a_{2}(n, 3)+2 a_{4}(n, 3)+24 a_{6}(n, 3) .
\end{aligned}
$$

\section{Summary}

We have presented expressions that describe the differences between low- $l$ p-mode frequencies extracted from full-disc, "Sunas-a-star" and resolved-Sun observations. These are given in terms of the even- $a_{j}$ coefficients used to describe the expansion of all $2 l+1$ components detected by resolved-Sun observations. Only $l+1$ of these are seen clearly in Sun-as-a-star data.

In all cases the basic resolved-data frequency used was the offset term in the expansion, $v_{n, l}^{\mathrm{RES}}$. This is often called the central frequency of the multiplet (i.e., its centroid).

Two classes of frequency extracted from full-disc, Sunas-a-star data were considered. First, that given by the "traditional" fitting approach, $v_{n, l}^{\mathrm{FDtrad}}$, in which the components within a multiplet are assumed to be distributed evenly in frequency. And second, from a model in which the components can be distributed unevenly as is observed in the presence of strong, non-homogeneous surface activity. The estimate thereby extracted, $v_{n, l}^{\mathrm{FDen}}$, is taken as the mean of the observable components. 


\section{Appendix A: Basis functions}

The following polynomials, $\mathcal{P}_{l}^{j}(m)$, are the basis functions used to parameterize the frequency-multiplet expansion for low- $l$, resolved observations (cf. Eq. (1)). In each case $L^{2}=l(l+1)$.

$\mathcal{P}_{l}^{1}(m)=m / l$

$\mathcal{P}_{l}^{2}(m)=\frac{6 m^{2}-2 L^{2}}{6 l^{2}-2 L^{2}}$

$\mathcal{P}_{l}^{3}(m)=\frac{20 m^{3}-4 m\left(3 L^{2}-1\right)}{20 l^{3}-4 l\left(3 L^{2}-1\right)}$,

$\mathcal{P}_{l}^{4}(m)=\frac{70 m^{4}-10 m^{2}\left(6 L^{2}-5\right)+6 L^{2}\left(L^{2}-2\right)}{70 l^{4}-10 l^{2}\left(6 L^{2}-5\right)+6 L^{2}\left(L^{2}-2\right)}$,

$$
\begin{gathered}
\mathcal{P}_{l}^{5}(m)=\frac{252 m^{5}-140 m^{3}\left(2 L^{2}-3\right)}{252 l^{5}-140 l^{3}\left(2 L^{2}-3\right)+l\left(20 L^{2}\left(3 L^{2}-10\right)+48\right)} \\
+\frac{m\left(20 L^{2}\left(3 L^{2}-10\right)+48\right)}{252 l^{5}-140 l^{3}\left(2 L^{2}-3\right)+l\left(20 L^{2}\left(3 L^{2}-10\right)+48\right)},
\end{gathered}
$$

and

$$
\begin{aligned}
\mathcal{P}_{l}^{6}(m)= & {\left[924 m^{6}-420 m^{4}\left(3 L^{2}-7\right)+84 m^{2}\left(5 L^{4}-25 L^{2}+14\right)\right.} \\
& \left.-20 L^{2}\left(L^{4}-8 L^{2}+12\right)\right] /\left[924 l^{6}-420 l^{4}\left(3 L^{2}-7\right)\right. \\
& \left.+84 l^{2}\left(5 L^{4}-25 L^{2}+14\right)-20 L^{2}\left(L^{4}-8 L^{2}+12\right)\right] .
\end{aligned}
$$

\section{References}

Aindow, A., Elsworth, Y. P., Isaak, G. R., et al. 1988, in Seismology of the Sun and Sun-like stars, ed. E. J. Rolfe, ESA-SP, 286, 157 Antia, H. M., Basu, S., Hill, F., et al. 2001, MNRAS, 327, 1029
Appourchaux, T., Toutain, T., Telljohann, U., et al. 1995, A\&A, 294, 13

Appourchaux, T., Anderson, B., \& Sekii, T. 2002, in SOHO 11 Symp. from Solar Min to Max: Half a Solar Cycle with SOHO, ed. C. Fröhlich, \& A. Wilson, ESA SP-508, 47

Basu, S., Christensen-Dalsgaard, J., Chaplin, W. J., et al. 1997, MNRAS, 292, 243

Basu, S., Turck-Chieze, S., Berthomieu, G., et al. 2000, ApJ, 535, 1078

Chaplin, W. J., \& Appourchaux, T. 1999, MNRAS, 309, 761

Chaplin, W. J., Elsworth, Y., Howe, R., et al. 1996, Sol. Phys., 168, 1

Chaplin, W. J., Elsworth, Y., Isaak, G. R., et al. 2003, MNRAS, 343, 343

Chaplin, W. J., Appourchaux, T., Elsworth, Y., et al. 2004, A\&A, 416, 341

Christensen-Dalsgaard, J., Däppen, W., Dziembowski, W. A., \& Guzik, J. A. 2000, in Variable Stars as Essential Astrophysical Tools, Proc. NATO Advanced Study Institute Held, Cesme, Turkey, ed. C. Ibanoglu (Kluwer Academic Publishers), 59

Christensen-Dalsgaard, J. 2002, Rev. Mod. Phys., 74, 1073

Couvidat, S., Turck-Chieze, S., \& Kosovichev, A. G. 2003, ApJ, 599, 1434

Duvall, T. L., Jefferies, S. M., Harvey, J. W., Osaki, Y., \& Pomerantz, M. A. 1993, ApJ, 410, 829

Dziembowski, W. A., Goode, P. R., Kosovichev, A. G., \& Schou, J. 2000, ApJ, 537, 1026

Fröhlich, C., Andersen, B. N., Appourchaux, T., et al. 1997, Sol. Phys., 170,1

Gabriel, A. H., Grec, G., Charra, J., et al. 1995, Sol. Phys., 162, 61

Harvey, J. W., Hill, F., Hubband, R., et al. 1996, Science, 272, 1284

Howe, R., Komm, R., \& Hill, F. 2002, ApJ, 580, 1172

Howe, R., Chaplin, W. J., Elsworth, Y., et al. 2003, ApJ, 588, 1204

Ritzwoller, M. H., \& Lavely, E. M. 1991, ApJ, 369, 557

Scherrer, P. H., Bogart, R. S., Bush, R. I., et al. 1995, Sol. Phys., 162, 129

Tomczyk, S., Streander, K., Card, G., et al. 1995, Sol. Phys., 159, 1

Toutain, T., Appourchaux, T., Baudin, F., et al. 1997, Sol. Phys., 175, 311

Toutain, T., Appourchaux, T., Fröhlich, C., et al. 1998, ApJ, 506, L147 